\title{
Latest Research Progress of the Pathogenesis of Depression and Its Status of TCM Treatment
}

\author{
Hesheng Zheng', Yumei $\mathrm{Wu}^{2, *}$ \\ ${ }^{1}$ Shaanxi University of Traditional Chinese Medicine, Xianyang, Shaanxi, China. \\ ${ }^{2}$ Fourth Military Medical University, Xi'an, Shaanxi, China.
}

How to cite this paper: Hesheng Zheng, Yumei Wu. (2022) Latest Research Progress of the Pathogenesis of Depression and Its Status of TCM Treatment. International Journal of Clinical and Experimental Medicine Research, 6(1), 97-102.

DOI: 10.26855/ijcemr.2022.01.016

Received: January 4, 2022

Accepted: January 28, 2022

Published: February 10, 2022

*Corresponding author: Yumei Wu, Fourth Military Medical University, Xi'an, Shaanxi, China.

Email: yumeiwu@fmmu.edu.cn

\begin{abstract}
Depressive disorder (DD) is one of the most common mental disorders and one of the main causes of disability in the world. Chronic or early life stress events are one of the main factors of DD. Recent studies have shown that the microbiota-gut-brain axis imbalance, the abnormal electrical activity of lateral habenula, and genetic variation are closely related to DD. Especially, in recent years, the emergence of the microbiota-gut-brain axis has attracted extensive attention. At present, the existing antidepressants and the therapeutic effect is not obvious even invalid for many patients, and it is likely to cause adverse effects. Traditional Chinese Medicine has obvious therapeutic effects, which are safe, reliable, low price, and significantly reduced the use of western medicine and its side effects. Therefore, traditional Chinese medicine and its therapeutic method gradually are applied to the clinical treatment of DD and achieved good results. The article summarizes the latest pathogenesis of DD and reviewed the mechanism of traditional Chinese medicine participating in DD treatment, to provide a reference for the clinical treatment of depression.
\end{abstract}

\section{Keywords}

Depressive Disorder, Microbiota-Gut-Brain Axis, Lateral Habenular Nucleus, Gene Mutation, Traditional Chinese Medicine

\section{Introduction}

Depressive disorder(DD) is a common mental disorder, along with depression, slow thinking, anhedonia, cognitive impairment, and sleep disorders, and even suicide when serious, which has a profound impact on the patient's physical health and quality of life and greatly increases the burden on family and social economy [1]. There were many causes of DD, such as inflammatory reaction, stressful life events, the disorder of hypothalamic-pituitary-adrenal (HPA) axis, the neuron cluster discharge of lateral habenular nucleus (LHb) and the gene mutation are closely related to DD [2-10]. In recent years, the pathogenesis and potential therapeutic methods of DD have been gradually discovered by researchers, which is particularly important for the improved quality of life of DD patients and reduction of social burden. To date, norepinephrine (NE) reuptake inhibitors and ketamine have obvious effects on the treatment of most DD patients but are also accompanied by obvious side effects. TCM has obvious therapeutic effects, is safe, reliable, and low price, and can significantly reduce the use of western medicine and its side effects. Therefore, more and more become an important choice for clinicians and patients.

$\mathrm{DD}$ is a disease that is highly related to life stress events. With the social economy entering a stage of rapid development, people's life pressure is increasing and there are more and more stressful events in social life, so the number of DD patients is bound to increase rapidly. Therefore, DD has become an influence on the health level and 
life quality of Chinese people.

This paper reviews the main mechanism of DD and enumerates the evidence of TCM participating in DD treatment in recent years, providing a reference for the clinical application of TCM in DD treatment.

\section{The role of microbiota-gut-brain axis in DD pathogenesis}

The intestinal microbiota is an extremely important part of the mammalian microbiome. The health and disease of the host will affect the status of intestinal microbiota, and the status of the intestinal microbiota can also affect the health and disease of the host. In addition, the intestinal microbiota plays an important role in the connection between the intestinal tract and the Central Nervous System (CNS). The gut-brain axis is an information channel for the bidirectional connection between the mammalian brain and gut, which is connected by the nerve, HPA axis, and the immune system [11, 12]. Research shows that many system functions are closely related to the gut-brain axis in the human body, including metabolic system, immune system, endocrine system, and nervous system, etc., and the exception of intestinal microbiota and gut-brain axis dysfunction affects brain function and control the behavior of the brain changes will also affect gut-brain axis structure and function of the, it is a two-way signal exchange mechanism [13]. It has been reported that intestinal microbiota directly affects the development and health of the mammalian brain [14], and the diversity and richness of intestinal microbiota in depressed patients are different from that in healthy people [15]. Researchers speculated that the rapid increase in the incidence of DD may be largely related to the widespread use of antibiotics in the past decades. Because antibiotics in the treatment of infection will also kill the symbiotic bacteria in the intestinal microbiota, resulting in the disorder of human microbes, which leads to the occurrence of psychiatric diseases [16-18]. Therefore, there may be a causal link between the disorder of intestinal microbiota and the occurrence of DD [15]. In addition, many patients with digestive system diseases are usually accompanied by mental diseases such as depression and anxiety, and their digestive diseases can be alleviated to some extent after psychiatric drug treatment. Therefore, people gradually realize that intestinal microbiota may play a key role in the pathogenesis of DD. Clinical studies have shown that there are significant differences in the intestinal microbiota of DD patients and healthy people, and the diversity and richness of intestinal microbiota of depressed patients are significantly decreased [19, 20]. In addition, various depressed animal models did show differences in intestinal microbiota between the control group and depressed animals, and there were similarities in intestinal microbiota between depressed animals and depressed patients [21-25]. Stressful life events are the main cause of DD. Studies have proved that chronic stress can interfere with the status of intestinal microbiota, cause the disorder of gut-brain axis function, and lead to the reduction of 5-HT content and brain-derived neurotrophic factor (BDNF) mRNA expression in the hippocampus, decreasing the level of plasma stress hormone, increases the level of interleukin-10 (IL-10) in circulating blood, leading to the occurrence of DD [21]. There is evidence that the brain can influence gut microbiota status through digestive and immune molecules, while the gut can also influence brain function through vagus and other mechanisms [26, 27]. The immune system is fundamental to survival in all species and plays a key role in regulating communication between gut microbiota and the brain, it has been shown that intestinal microbiota can release various immune agonists [such as lipopolysaccharide (LPS) and peptidoglycan, etc.] to ensure the normal operation of the immune system function. LPS and peptidoglycan immune agonist entered the blood can accompany the circulation of the blood by the blood-brain-barrier into the brain [28], and the NE and LPS can induce the depression behavior of mice, and widely used in depression model mice, so the NE and LPS relate to DD Close relationship. In addition, the intestinal microbiota of DD model rats can promote the inflammatory process of the ventral hippocampus (such as enhanced microglial cell activity, increased IL-1 $\beta$ level and increased blood-brain barrier permeability, etc.), suggesting that intestinal microbiota may induce depressive-like behavior in mice by promoting the inflammatory response of ventral hippocampus [29]. Therefore, the microbiota-gut-brain axis may mediate DD through the immune mechanism of intestinal microbiota metabolites and the inflammatory response promoting the ventral hippocampus, but the specific mechanism is not clear.

\section{The role of abnormal electrical activity in lateral habenula in DD}

With the deepening of researches on the pathogenesis of DD, more and more new mechanisms have been discovered and gradually applied to the development of antidepressant therapy and targeted antidepressants. Studies have shown that abnormal electrical activity of LHb is closely related to the occurrence of DD [30-34]. LHb is a brain structure with good phylogeny and preservation, and a key region for regulating communication between forebrain, midbrain, and hindbrain monoaminergic systems [35-37]. Studies have shown that LHb mediates DD 
through a special discharge mode (cluster discharge), and the role of the antidepressant ketamine is to block this cluster discharge of LHb [38], thus playing a treatment effect on DD. In addition, it was proved that the potassium channel Kir4.1 in LHb astrocytes triggered the cluster discharge of its neurons, which ultimately lead to the occurrence of depression-like behavior [39]. This was the first time in DD research to reveal the molecular mechanism related to LHb and depression, and to find a new target for rapid treatment of DD.

\section{The role of genetic variation in DD}

Genetic factors are also a non-negligible part of DD incidence. Genome-wide association analysis found 102 genetic variations related to DD and emphasized the importance of the prefrontal cortex in genetic variations of DD [6]. Astrocytes have been shown to produce pro-inflammatory cytokines such as IL-1 $\beta$, and nuclear factor- $\mathrm{KB}(\mathrm{NF}-\mathrm{\kappa B})$, a pro-inflammatory transcription activator, is a key regulator of CNS inflammation [40-42]. In addition, MEN1 was found to be a novel DD pathogenic gene. MEN1 gene mutation blocks the binding of multiple endocrine tumor protein (MENIN) and NF-kB-P65, leading to the overactivation of NF- $\mathrm{BB}$ and IL-1 $\beta$ pathways, leading to CNS neuroinflammation, and subsequently to depression-like behavior [4]. These research results at the gene level will greatly promote people's understanding of the underlying genetic mechanism of DD, provide a new approach for the study of depression mechanism, and provide a new target and direction for DD treatment.

\section{The status of DD treatment with Traditional Chinese medicine}

DD is the name of western medicine, and TCM believes that DD belongs to the category of depression syndrome, belonging to "emotional disease", which is scattered in ancient medical books such as "lily disease", "mania", "hysteria", "emotional disease", etc. Currently, clinical diagnosis and treatment are mostly based on "emotional disease". Drug treatment is the main therapeutic method for DD 5-HT and NE uptake inhibitors, tricyclic antidepressants, monoamine oxidase inhibitors, atypical antidepressants, and other antidepressants. Although these antidepressants have obtained certain effects in the clinical treatment, but there are some shortcomings, such as the efficiency is low, working slowly, adverse reactions. A decrease in western medicine and TCM for consumption improves the efficiency of western medicine and plays an important role in reducing the incidence of adverse reactions. Therefore, more and more clinicians apply TCM to treatment DD.

\subsection{Application of TCM monomer in DD treatment}

The monomer of TCM is the active ingredient extracted from TCM and has shown antidepressant effects in various DD animal models. Studies have shown that ginsenoside Rg1 can significantly improve depression-like behavior induced by chronic unpredictable mild stress (CUMS) in rats, reduce oxidative stress and inflammatory response in the hippocampus CA1 region of rats, and reduce apoptosis in this region [43]. In addition, resveratrol significantly reduced the expression level of pro-inflammatory cytokines and up-regulated the expression of BDNF in the prefrontal cortex (PFC) and hippocampus in depressed mice [44]. It has also been reported that paeoniflorin can increase the density of dendritic spines of CA1 neurons in depressed mice, inhibit the activation of microglia cells after hippocampal injury, and effectively improve depression-like behavior induced by reserpine in mice [45]. Moreover, bacopin extract can adjust the intestinal microbiota diversity and improve the abundance of bifidobacteria that effecting the metabolism of tryptophan, and inhibition of tryptophan-dog urine pathway to decrease metabolites wich uric acid, amino acid and reduce the brain dog urine metabolites of quinoline acid amino acid generated, thereby improving the depression in mice induced by LPS [46].

\subsection{Application of TCM compound in DD treatment}

TCM compound refers to a prescription consisting of two or more TCM. It is one of the most widely used TCM therapeutic methods in the clinic and is also widely used in the prevention and treatment of CNS diseases. Studies have found that Chaihu Shugan San can increase the expression of BDNF in the hippocampus and PFC and enhance the level of adrenocorticotropin-releasing hormone in plasma, and reduce the content of 5-HT and DA in plasma, thus alleviating cumS induced depressive behavior in rats [47, 48]. In addition, Chaihu Shugan Pill can inhibit neuronal apoptosis in the hippocampus by regulating the expression of genes with opposite Bax and Bcl-2 functions, thereby increasing autonomous activity and reducing immobile time in diabetic and depressed rats [49]. Studies have shown that Banxia Houpu decoction can reduce cumS induced depressive behavior in rats by reducing the activation of NLRP3 inflammasome in the liver and brain [50]. Suanzaoren decoction significantly decreased 
acidic protein (GFAP) and coloration 43 (Connexin 43, Cx43) expression levels, suggesting that suanzaoren decoction may have an antidepressant effect by reducing the damage of glial cells in the cerebral cortex [51].

\subsection{Application of acupuncture in DD treatment}

Acupuncture is an important part of TCM, is widely recognized by the whole world as a clinical treatment, and plays an important role in the treatment of CNS disorders. Research shows that the occurrence of DD and is closely related to HPA axis negative feedback mechanism disorder, and two acupuncture methods which are Tongdu tiaosheng and shugam jieyu can be ameliorated feel down in the dumps and lack of pleasure by adjusting HPA in depressed model rats [52]. In addition, acupuncture at Baihui point and Neiguan point can improve depressive-like behavior of DD model rats, and significantly down-regulate the levels of IL-6, iL-1 $\beta$, and TNF- $\alpha$ in serum and brain tissues [53]. Studies have shown that acupuncture treatment can cause changes in the activity of neurons in different brain regions of DD patients, and the corresponding brain signal changes affected by acupuncture at different acupoints are also different [54]. Mitogen-activated protein kinase (MAPK) signaling pathway is closely related to DD, and the C-Jun terminal kinase (JNK) signaling pathway is a member of the MAPK signaling pathway, which is mainly involved in the apoptosis process and plays an important role in the pathogenesis of DD. Moreover, studies have shown that acupuncture can play an anti-depressive effect by down-regulating the expression of JNK signaling pathway-related proteins in PFC [55-57]. Electroacupuncture can significantly change the content of gastrin (GAS), neuropeptide Y (NPY), and calcitonin gene-related peptide in colon mucosa of depressed rats, and regulate the GAS, somatostatin in the hypothalamus of rats. The release of motilin regulates the gut-brain axis to improve the digestive function of depressed rats $[58,59]$. These results indicate that acupuncture treatment can improve intestinal function and treat DD by regulating intestinal microbiota and brain-gut peptide levels.

\section{Discussion}

Since always, it is widely believed that DD is associated with nerve regeneration, neural cell death, neuroinflammation, and neuroendocrine system. In recent years, research has shown that the microbiota-gut-brain axis imbalance, the abnormal electrical activity of lateral habenula, and genetic variation are closely related to DD. Clinical practices have confirmed that TCM treatment is a safe, effective, less adverse reaction, cheap therapeutic method, was gradually applied to the prevention and treatment of CNS diseases and has obtained a good effect in the treatment of DD. Treatment of TCM reduces the dosage of western medicine and improves efficiency while reducing the incidence of adverse reactions of western medicine. Therefore, to explore the involved in the mechanism of action of DD treatment of TCM for treatment of DD is particularly important.

\section{References}

[1] Pereira, J. D. C., Rea, K., Nolan, Y. M., et al. (2020). Depression’s Unholy Trinity: Dysregulated Stress, Immunity, and the Microbiome [J]. Annual Review of Psychology, 2020, 71(1): null.

[2] Cui, Y., Hu, S., Hu, H. (2019). Lateral Habenular Burst Firing as a Target of the Rapid Antidepressant Effects of Ketamine [J]. Trends Neurosci, 2019, 42(3): 179-191.

[3] Ng, A., Tam, W. W., Zhang, M. W., et al. (2018). IL-1beta, IL-6, TNF- alpha and CRP in Elderly Patients with Depression or Alzheimer's disease: Systematic Review and Meta-Analysis [J]. Sci Rep, 2018, 8(1): 12050.

[4] Leng, L., Zhuang, K., Liu, Z., et al. (2018). Menin deficiency leads to depressive-like behaviors in mice by modulating astrocyte-mediated neuroinflammation [J]. Neuron, 2018, 100(3): 551-563. e557.

[5] De Kloet, E. R., Joëls, M., Holsboer, F. (2005). Stress and the brain: from adaptation to disease [J]. Nature reviews neuroscience, 2005, 6(6): 463.

[6] Howard, D. M., Adams, M. J., Clarke, T.-K., et al. (2019). Genome-wide meta-analysis of depression identifies 102 independent variants and highlights the importance of the prefrontal brain regions [J]. Nature Neuroscience, 2019, 22(3): 343.

[7] Evrensel, A., Ceylan, M. E. (2015). The Gut-Brain Axis: The Missing Link in Depression [J]. Clin Psychopharmacol Neurosci, 2015, 13(3): 239-244.

[8] Hammen, C. (2016). Depression and stressful environments: identifying gaps in conceptualization and measurement [J]. Anxiety Stress Coping, 2016, 29(4): 335-351.

[9] Gotlib, I. H., Joormann, J. (2010). Cognition and depression: current status and future directions [J]. Annu Rev Clin Psychol., 2010, 6: 285-312. 
[10] Turner, R. J., Wheaton, B., Lloyd, D. A. (1995). The epidemiology of social stress [J]. American Sociological Review, 1995: 104-125.

[11] Forsythe, P., Sudo, N., Dinan, T., et al. (2010). Mood and gut feelings [J]. Brain, Behavior, and Immunity, 2010, 24(1): 9-16.

[12] Liang, S., Wang, T., Hu, X., et al. (2012). Microorganism and behavior and psychiatric disorders [J]. Advances in Psychological Science, 2012, 20(1): 75-97.

[13] Liang, S., Wu, X., Hu, X., et al. (2018). Recognizing depression from the microbiota-gut-brain axis [J]. International Journal of Molecular Sciences, 2018, 19(6): 1592.

[14] Evrensel, A., Önen Ünsalver, B., Ceylan, M. E. (2019). Therapeutic potential of the microbiome in the treatment of neuropsychiatric disorders [J]. Medical Sciences, 2019, 7(2): 21.

[15] Evrensel, A., Unsalver, B. O., Ceylan, M. E. (2019). Neuroinflammation, Gut-Brain Axis and Depression [J]. Psychiatry Investig, 2019.

[16] Bercik, P., Collins, S. M. (2014). The effects of inflammation, infection and antibiotics on the microbiota-gut-brain axis [A]. In: microbial endocrinology: the microbiota-gut-brain axis in health and disease: Springer, 2014: 279-289.

[17] Lurie, I., Yang, Y.-X., Haynes, K., et al. (2015). Antibiotic exposure and the risk for depression, anxiety, or psychosis: a nested case-control study [J]. The Journal of Clinical Psychiatry, 2015, 76(11): 1522-1528.

[18] Fröhlich, E. E., Farzi, A., Mayerhofer, R., et al. (2016). Cognitive impairment by antibiotic-induced gut dysbiosis: analysis of gut microbiota-brain communication [J]. Brain, Behavior, and Immunity, 2016, 56: 140-155.

[19] Kelly, J. R., Borre, Y., O'Brien, C., et al. (2016). Transferring the blues: depression-associated gut microbiota induces neurobehavioural changes in the rat [J]. Journal of Psychiatric Research, 2016, 82: 109-118.

[20] Jiang, H., Ling, Z., Zhang, Y., et al. (2015). Altered fecal microbiota composition in patients with major depressive disorder [J]. Brain, Behavior, and Immunity, 2015, 48: 186-194.

[21] Liang, S., Wang, T., Hu, X., et al. (2015). Administration of Lactobacillus helveticus NS8 improves behavioral, cognitive, and biochemical aberrations caused by chronic restraint stress [J]. Neuroscience, 2015, 310: 561-577.

[22] Park, A., Collins, J., Blennerhassett, P., et al. (2013). Altered colonic function and microbiota profile in a mouse model of chronic depression [J]. Neurogastroenterology \& Motility, 2013, 25(9): 733-e575.

[23] Yu, M., Jia, H., Zhou, C., et al. (2017). Variations in gut microbiota and fecal metabolic phenotype associated with depression by 16S rRNA gene sequencing and LC/MS-based metabolomics [J]. Journal of Pharmaceutical and Biomedical Analysis, 2017, 138: 231-239.

[24] Bharwani, A., Mian, M. F., Surette, M. G., et al. (2017). Oral treatment with Lactobacillus rhamnosus attenuates behavioural deficits and immune changes in chronic social stress [J]. BMC Medicine, 2017, 15(1): 7.

[25] O'Mahony, S. M., Marchesi, J. R., Scully, P., et al. (2009). Early life stress alters behavior, immunity, and microbiota in rats: implications for irritable bowel syndrome and psychiatric illnesses [J]. Biological Psychiatry, 2009, 65(3): 263-267.

[26] Foster, J. A., Neufeld, K.-A. M. (2013). Gut-brain axis: how the microbiome influences anxiety and depression [J]. Trends in Neurosciences, 2013, 36(5): 305-312.

[27] Bravo, J. A., Forsythe, P., Chew, M. V., et al. (2011). Ingestion of Lactobacillus strain regulates emotional behavior and central GABA receptor expression in a mouse via the vagus nerve [J]. Proceedings of the National Academy of Sciences, 2011, 108(38): 16050-16055.

[28] Sherwin, E., Bordenstein, S. R., Quinn, J. L., et al. (2019). Microbiota and the social brain [J]. Science, 2019, 366(6465).

[29] Pearson-Leary, J., Zhao, C., Bittinger, K., et al. (2019). The gut microbiome regulates the increases in depressive-type behaviors and in inflammatory processes in the ventral hippocampus of stress vulnerable rats [J]. Molecular Psychiatry, $2019: 1$.

[30] Li, K., Zhou, T., Liao, L., et al. (2013). BCaMKII in lateral habenula mediates core symptoms of depression [J]. Science, 2013, 341(6149): 1016-1020.

[31] Morris, J., Smith, K., Cowen, P., et al. (1999). Covariation of activity in habenula and dorsal raphe nuclei following tryptophan depletion [J]. Neuroimage, 1999, 10(2): 163-172.

[32] Lecca, S., Pelosi, A., Tchenio, A., et al. (2016). Rescue of GABA B and GIRK function in the lateral habenula by protein phosphatase 2A inhibition ameliorates depression-like phenotypes in mice [J]. Nature Medicine, 2016, 22(3): 254.

[33] Li, B., Piriz, J., Mirrione, M., et al. (2011). Synaptic potentiation onto habenula neurons in the learned helplessness model of depression [J]. Nature, 2011, 470(7335): 535.

[34] Shumake, J., Edwards, E., Gonzalez-Lima, F. (2003). Opposite metabolic changes in the habenula and ventral tegmental area of a genetic model of helpless behavior [J]. Brain Research, 2003, 963(1-2): 274-281.

[35] Herkenham, M., Nauta, W. J. (1979). Efferent connections of the habenular nuclei in the rat [J]. Journal of Comparative Neurology, 1979, 187(1): 19-47. 
[36] Herkenham, M. (1979). The afferent and efferent connections of the ventromedial thalamic nucleus in the rat [J]. Journal of Comparative Neurology, 1979, 183(3): 487-517.

[37] Aizawa, H., Amo, R., Okamoto, H. (2011). Phylogeny and ontogeny of the habenular structure [J]. Frontiers in Neuroscience, 2011, 5: 138.

[38] Yang, Y., Cui, Y., Sang, K., et al. (2018). Ketamine blocks bursting in the lateral habenula to rapidly relieve depression [J]. Nature, 2018, 554(7692): 317-322.

[39] Cui, Y., Yang, Y., Ni, Z., et al. (2018). Astroglial Kir4.1 in the lateral habenula drives neuronal bursts in depression [J]. Nature, 2018, 554(7692): 323-327.

[40] Norman, G., Karelina, K., Zhang, N., et al. (2010). Stress and IL-1 $\beta$ contribute to the development of depressive-like behavior following peripheral nerve injury [J]. Molecular psychiatry, 2010, 15(4): 404.

[41] Koo, J. W., Duman, R. S. (2009). Evidence for IL-1 receptor blockade as a therapeutic strategy for the treatment of depression [J]. Current opinion in investigational drugs (London, England: 2000), 2009, 10(7): 664.

[42] Oeckinghaus, A., Ghosh, S. (2009). The NF- $\mathrm{B}$ family of transcription factors and its regulation [J]. Cold Spring Harbor perspectives in biology, 2009, 1(4): a000034.

[43] Y. Li, L. Wang, P. Wang, et al. (2020). Ginsenoside-Rg1 Rescues Stress-Induced Depression-Like Behaviors via Suppression of Oxidative Stress and Neural Inflammation in Rats [J]. Oxid Med Cell Longev, 2020, 2020: 2325391.

[44] L. Ge, L. Liu, H. Liu, et al. (2015). Resveratrol abrogates lipopolysaccharide-induced depressive-like behavior, neuroinflammatory response, and CREB/BDNF signaling in mice [J]. Eur J Pharmacol., 2015, 768: 49-57.

[45] D. D. Tian, M. Wang, A. Liu, et al. (2021). Antidepressant Effect of Paeoniflorin Is Through Inhibiting Pyroptosis CASP-11/GSDMD Pathway [J]. Mol Neurobiol, 2021, 58 (2): 761-776.

[46] W. J. Fu. Bacopin can regulate intestinal microbiota to affect KP pathway and improve depression in mice [D]. China Medical University, 2021.

[47] L. Yan, X. Xu, Z. He, et al. (2020). Antidepressant-Like Effects and Cognitive Enhancement of Coadministration of Chaihu Shugan San and Fluoxetine: Dependent on the BDNF-ERK-CREB Signaling Pathway in the Hippocampus and Frontal Cortex [J]. Biomed Res Int, 2020, 2020: 2794263.

[48] Y. Liu, W. Wang, Y. Chen, et al. (2020). Simultaneous quantification of nine components in the plasma of depressed rats after oral administration of Chaihu-Shugan-San by ultra-performance liquid chromatography/quadrupole-time-of-flight mass spectrometry and its application to pharmacokinetic studies [J]. J Pharm Biomed Anal., 2020, 186: 113310.

[49] S. S. Peng, J. Yue. (2018). Effects of Chaihu Shugan pill on behavioral performance and cognitive function in diabetic rats with depression [J]. Chinese Journal of Gerontology, 2018, 38(24): 6069-6071.

[50] K. K. Jia, Y. J. Zheng, Y. X. Zhang, et al. (2017). Banxia-houpu decoction restores glucose intolerance in CUMS rats through improvement of insulin signaling and suppression of NLRP3 inflammasome activation in liver and brain [J]. J Ethnopharmacol, 2017, 209: 219-229.

[51] K. H. Liu, J. W. Sun, X. H. Hu. (2019). Effect of Suanzaoren Decoction on glial fibrillary acidic protein and gap junction protein 43 in cerebral cortex astrocytes of depressed rats [J]. New Traditional Chinese Medicine, 2019, 51(10): 13-16.

[52] X. J. Song, R. Li, N. Ding, et al. (2016). Effects of tongdu tiaosheng and shugan jieyu on behavior and HPA axis of depression model rats [J]. Journal of Clinical Acupuncture and Moxibustion, 2016, 32(02): 64-68.

[53] S. Y. Jin, L. Hu, W. Y. Bao, et al. (2014). Effects of acupuncture on serum and encephalitis cytokines in chronic stress depressed rats [J]. Journal of Clinical Acupuncture and Moxibustion, 2014, 30(05): 57-60.

[54] Y. Yi, F. M. Xu, P. Xie, et al. (2012). Resting state functional magnetic resonance study of acupuncture Taichong acupoint regulating brain function in depression [J]. China Journal of Traditional Chinese Medicine and Pharmacy, 2012, 27(02): 369-373.

[55] T. B. Brust, F. S. Cayabyab, and B. A. MacVicar. (2007). C-Jun N-terminal kinase regulates adenosine A1 receptor-mediated synaptic depression in the rat hippocampus [J]. Neuropharmacology, 2007, 53 (8): 906-17.

[56] M. Mitic, I. Lukic, N. Bozovic, et al. (2015). Fluoxetine signature on hippocampal MAPK signalling in sex-dependent manner [J]. J Mol Neurosci, 2015, 55(2): 335-46.

[57] Q. Y. Yu, X. J. Yang, H. L. Jiang, et al. (2021). Effect of acupuncture on JNK signaling pathway expression in prefrontal cortex of depressed rats [J]. China Journal of Traditional Chinese Medicine and Pharmacy, 2021, 36(06): 3157-3161.

[58] J. Y. Teng, Z. G. Li, Y. Bai, et al. (2013). Effects of different electroacupuncture on the content of GAS NPY CGRP in colonic mucosa of depressed rats [J]. World Journal of Integrated Traditional and Western Medicine, 2013, 8(03): 226-229.

[59] L. N. Qin. (2007). Study on the mechanism of electroacupuncture improving digestive function in depressed rats [D]. Beijing University of Traditional Chinese Medicine, 2007. 